\title{
Interpenetrated Spherulites of Poly(butylene succinate)/ Poly(vinylidene chloride-co-vinyl chloride) Blends. An Optical Microscopic Study
}

\author{
Takayuki IKEHARA and Toshio NISHI \\ Department of Applied Physics, School of Engineering, The University of Tokyo, \\ 7-3-1, Hongo, Bunkyo-ku, Tokyo 113-8656, Japan
}

(Received February 4, 2000)

\begin{abstract}
Interpenetrated spherulites of poly(butylene succinate)/poly(vinylidene chloride-co-vinyl chloride) (PBSU/PVDCVC) blends were investigated by optical microscopy. The spherulites of PBSU and PVDCVC were negative and positive respectively, and birefringence of PVDCVC was small under crossed nicols. The spherulitic growth rate of PVDCVC showed complex dependence on the crystallization temperature $T_{\mathrm{c}}$. Interpenetrated spherulites were formed under most of the experimental conditions, PBSU content $30 \%-60 \%$ and $T_{\mathrm{c}} 338 \mathrm{~K}-378 \mathrm{~K}$. Optical microscopy revealed that the birefringent pattern of the area of a PVDCVC spherulite changed into that of a PBSU spherulite after penetration. This result indicates that the lamellae of PBSU grow along those of PVDCVC, and it is the evidence for the formation of interpenetrated spherulites. The microscopic observations implied that the density of lamellae in PVDCVC was sparse. The birefringent pattern of PBSU spherulites changed with experimental conditions. The blends with low PBSU content showed neither negative nor positive spherulite. This birefringence was also kept in the area of PVDCVC spherulites after penetration.
\end{abstract}

KEY WORDS Poly(butylene succinate)/Poly(vinylidene chloride-co-vinyl chloride)/Interpenetrated Spherulites / Birefringence /

Poly(butylene succinate)/poly(vinylidene chloride-covinyl chloride) (PBSU/PVDCVC) blends are a miscible crystalline/crystalline polymer system above the melting points. They were found to show interpenetrated (or interlocked) spherulites, ${ }^{1}$ which do not stop growing when they collide with neighboring spherulites. An important factor to realize interpenetration is the simultaneous growth of the spherulites of the two constituents. The melting points $T_{\mathrm{m}}$ of PBSU and PVDCVC are $387 \mathrm{~K}$ and $421 \mathrm{~K}$, respectively. On the other hand, the spherulitic growth rate $G$ of PBSU, the lower- $T_{\mathrm{m}}$ component, is usually faster than that of PVDCVC, the higher- $T_{\mathrm{m}}$ component. This enables the crystallization of the lower- $T_{\mathrm{m}}$ component to influence that of the higher- $T_{\mathrm{m}}$ component in this system. PBSU/PVDCVC blends were discovered in a series of studies of polyester/poly(vinylidene halide) systems by our group. ${ }^{1 \cdots 3}$

Studies of miscible pairs of crystalline polymers with different chemical structures ${ }^{4-9}$ are still restricted compared with crystalline/amorphous and amorphous/amorphous blends. The reported $T_{\mathrm{m}}$ differences are usually about $100 \mathrm{~K}$ in these systems. The higher- $T_{\mathrm{m}}$ component crystallizes first in the crystallization process and the other component crystallizes in the crystals where it is specially constrained. Hence, the simultaneous growth of the spherulites of both components is hardly observed except the blends of the same kind of polymers such as low-density and high-density polyethylene. Our group has been seeking crystalline/crystalline polymer blends with different chemical structures exhibiting simultaneous spherulitic growth.

The purpose of the present paper is to investigate the dependence of $G$ on the crystallization temperature $T_{\mathrm{c}}$, the conditions of the formation of interpenetrated spherulites, and their morphology by optical microscopy.

\section{EXPERIMENTAL}

The characteristics of the samples are displayed in Table I. The constituent polymers were dissolved into a mutual solvent $N, N$-dimethylformamide at about $370 \mathrm{~K}$. Cast films were prepared on glass plates and they were dried in air before the removal of the residual solvent in a vacuum chamber for several days at room temperature. They were covered by other glass plates for optical microscopy.

The spherulitic growth was observed under crossed nicols with a polarizing microscope (Olympus BHA-P) and a temperature controller (Linkam LK-600PM). After melted at $443 \mathrm{~K}$ for $10 \mathrm{~min}$, the samples were quenched to $T_{\mathrm{c}}$ at $-100 \mathrm{~K} \mathrm{~min}^{-1}$. A test plate of $530 \mathrm{~nm}$ was used if necessary since small birefringence of PVDCVC makes optical microscopic observation difficult. The compositions of the blends were PBSU/PVDCVC (wt/wt) $=30 / 70$, $40 / 60,50 / 50$, and $60 / 40$. The experimental temperature range was $338 \mathrm{~K} \leq T_{\mathrm{c}} \leq 378 \mathrm{~K}$ at intervals of $5 \mathrm{~K}$.

\section{RESULTS}

As shown in Figure 1 neat PBSU showed bright, negative spherulites, and PVDCVC showed dark, positive spherulites. These differences make distinguishing the spherulites of the two polymers easy by optical micros-

Table I. The characteristics of the samples: source,weight average molecular weight $M_{w}$. glass transition temperature $T_{k}$, and melting temperature $T_{\mathrm{m}}$

\begin{tabular}{lcccc}
\hline Sample & \multicolumn{1}{c}{ Source } & $M_{w}$ & $T_{\mathrm{g}} / \mathrm{K}$ & $T_{\mathrm{m}} / \mathrm{K}$ \\
\hline PBSU & Showa Denko & 140000 & 241 & 387 \\
PVDCDVC $^{\text {a }}$ & Asahi Chemical & 100000 & 279 & 421 \\
\hline
\end{tabular}

"Vinylidene chloride/vinyl chloride $=80 / 20$. 

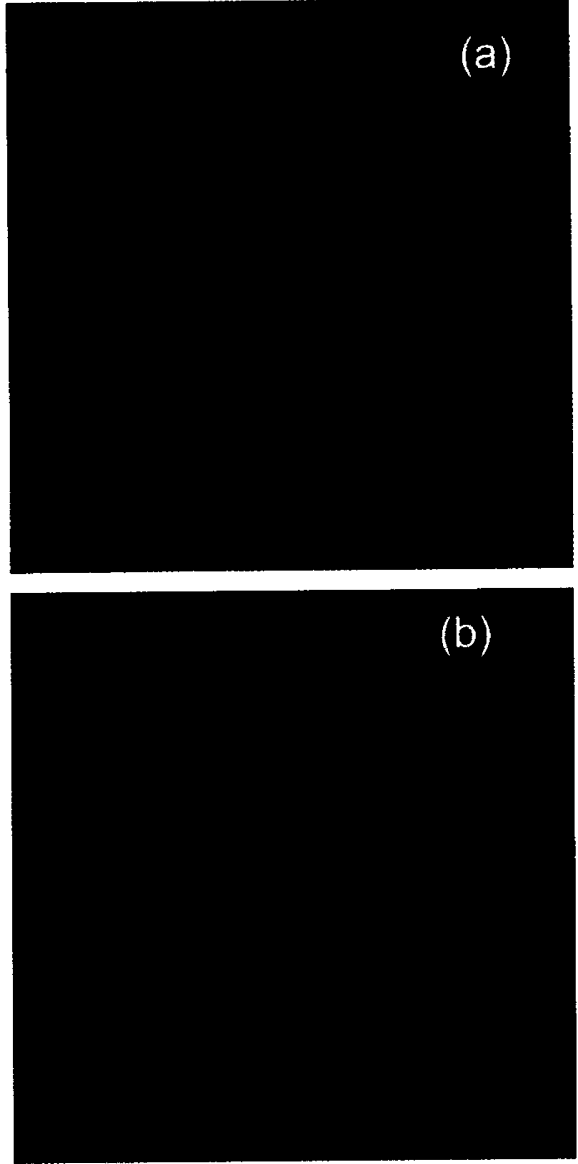

Figure 1. Spherulites of (a) PBSU crystallized at $363 \mathrm{~K}$ and (b) PVDCVC at $358 \mathrm{~K}$, which show negative and positive spherulites, respectively. Bar $50 \mu \mathrm{m}$.

copy. Table II summarizes the results of the spherulitic growth. Interpenetrated spherulites were observed under all the conditions where both constituents showed spherulitic growth. It should be noted that the spherulites of neat PBSU or PVDCVC never showed interpenetration by themselves.

Figure 2 shows the $T_{\mathrm{c}}$ dependence of $G$. Spherulites grew linearly until they collided with neighboring ones as in the previous work. ${ }^{1}$ PBSU and PVDCVC had almost the same values of $G$ in a $30 / 70$ blend. They were approximately the same at about $360 \mathrm{~K}, 370 \mathrm{~K}$, and 370 $\mathrm{K}$ for $40 / 60,50 / 50$, and $60 / 40$ blends, respectively. The blend composition influenced $G$ of PBSU, whereas it affected little that of PVDCVC. The peak temperature of $G$ of PVDCVC increased with the PBSU content, and the $G^{-} T_{\mathrm{c}}$ lines crossed around $360 \mathrm{~K}$.

The penetration process, which will be discussed later, is displayed in Figure 3. The bright spherulite of PBSU at the center (S1) collided with the dark spherulite of PVDCVC (S2) on the upper right in (a). The brightness of the area in $\mathrm{S} 2$ near the collision border gradually increased in (b) and the bright area expanded toward the center of S2. At the same time, S1 continued to grow around $\mathrm{S} 2$. In (c), the bright area in $\mathrm{S} 2$ passed the center of S2 and reached the opposite border of S2. Finally, in (d), S1 surrounded S2 and continued to grow. The birefringent pattern in the area of S2 was not the superposition of S1 and S2 after the penetration; the apparent
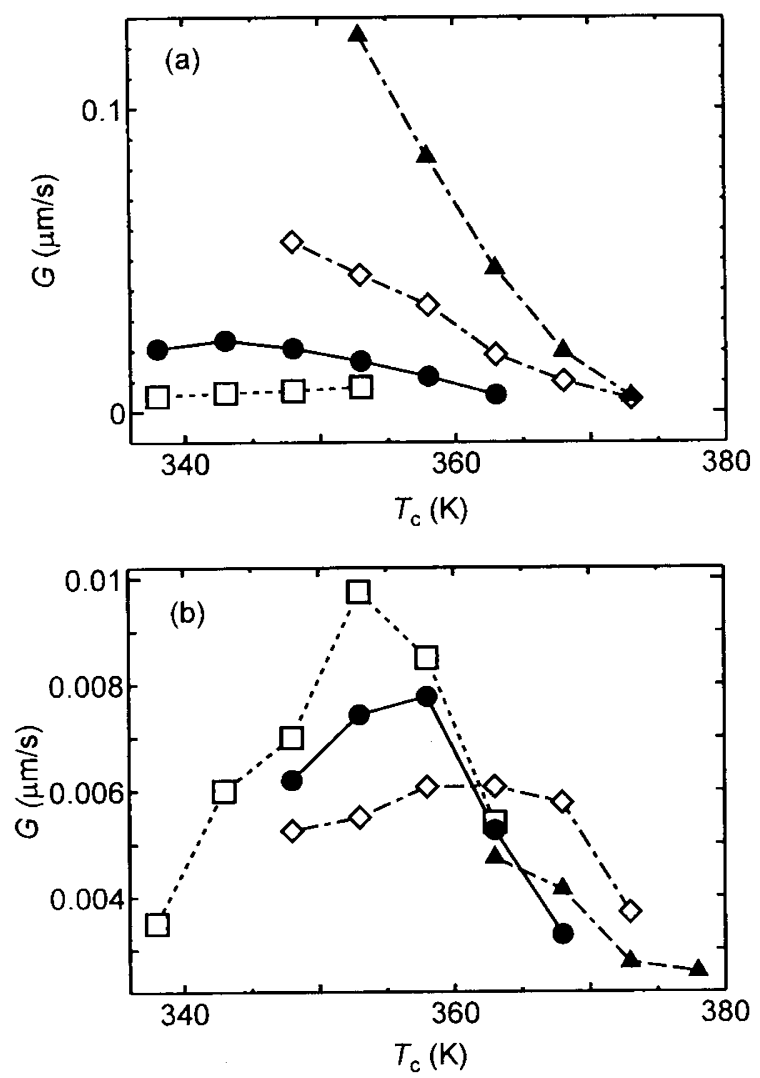

Figure 2. Crystallization temperature $T_{\mathrm{c}}$ dependence of the spherulitic growth rate $G$ of (a) PBSU and (b) PVDCVC. PBSU/ $\mathrm{PVDCVC}=30 / 70(\square), 40 / 60(\bigcirc), 50 / 50(\diamond)$, and $60 / 40(\mathbf{A})$.

Table II. The conditions for spherulitic growth. $\chi$ : both PBSU and PVDCVC showed spherulitic growth. $O$ :only PBSU spherulites were observed. $\times$ : only PVDCVC spherulites grew. - : no experiments were performed

\begin{tabular}{|c|c|c|c|c|c|c|c|c|c|}
\hline \multirow{2}{*}{ PBSU/PVDCVC } & \multicolumn{9}{|c|}{$T_{\mathrm{c}} / \mathrm{K}$} \\
\hline & 338 & 343 & 348 & 353 & 358 & 363 & 368 & 373 & 378 \\
\hline $30 / 70$ & $(\otimes)$ & $\otimes$ & $\otimes$ & $(\mathbb{X}$ & $\times$ & $x$ & - & - & - \\
\hline $40 / 60$ & 0 & 0 & $\otimes$ & $(x)$ & $\otimes$ & $\otimes$ & $\times$ & - & - \\
\hline $50 / 50$ & - & - & $\otimes$ & $\otimes$ & ( & X & $x$ & 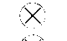 & - \\
\hline $60 / 40$ & - & - & - & 0 & $\bigcirc$ & 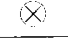 & $X$ & $x$ & $\times$ \\
\hline
\end{tabular}

pattern was that of a PBSU spherulite whose center is located at the center of S2. In Figure 3 (a), the size of S2 was the same as that of the PVDCVC spherulite close to the upper left corner of the photograph (S3). After S1 passed $\mathrm{S} 2$ in (d), the size of $\mathrm{S} 2$ was almost unchanged while S3 continued to grow.

PBSU spherulites showed three birefringent patterns under crossed nicols with a test plate according to the crystallization conditions as in Figure 4: (a) negative spherulites, (b) disordered near the center and negative in the outer regions, and (c) neither negative nor positive spherulites that rotated by about $45^{\circ}$ from the positive and negative patterns. The change of the birefringent pattern is summarized in Figure 5.

As exhibited in Figure 6, the birefringent pattern in penetrated PVDCVC spherulites was close to that of PBSU. The birefringent patterns of PBSU and PVDCVC spherulites were pattern (c) and positive, respectively, before penetration. After penetration, the area of 

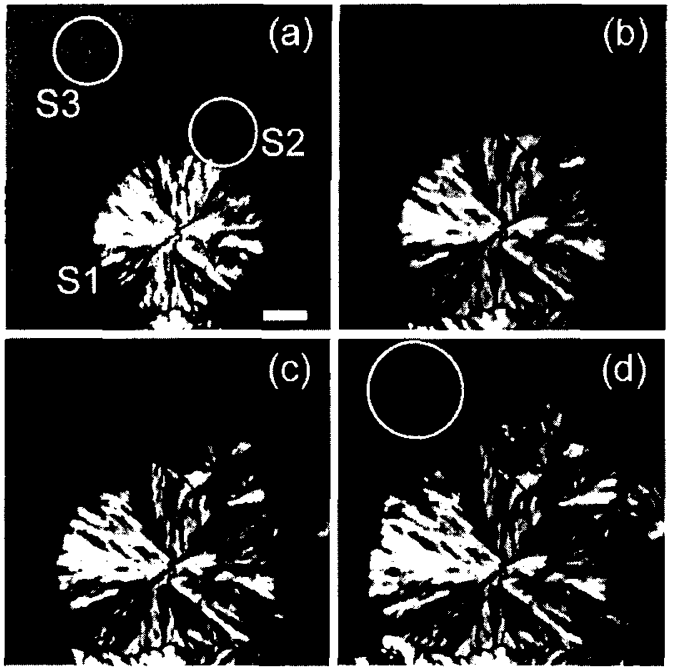

Figure 3. The process of interpenetration in a $40 / 60$ blend at 353 $\mathrm{K}$. The crystallization times are (a) $44 \mathrm{~min}$, (b) $55 \mathrm{~min}$, (c) $60 \mathrm{~min}$ and (d) $70 \mathrm{~min}$. The white lines indicate the positions of PVDCVC spherulites. S1 is a PBSU spherulite and S2 and S3 are PVDCVC spherulites. Bar $10 \mu \mathrm{m}$.

PVDCVC spherulites showed similar birefringence as PBSU.

\section{DISCUSSIONS}

As indicated in Figure 1, the spherulites of PVDCVC were darker than those of PBSU under crossed nicols. It shows that PBSU has larger birefringence than PVDCVC. The possible causes for this result are the differences of the thickness of the sample, the crystalline structure, the lamellar thickness, and the density of lamellae. The thickness of the sample is ruled out since it is almost the same for the spherulites of the two components growing simultaneously in blended samples. Though quantitative estimation of the film thickness was not performed, the film thickness must have been thin enough for the spherulites to contact with both upper and lower glass plates in all the photographs in this paper and to grow two-dimensionally.

The spherulitic growth of the two components was observed under almost all experimental conditions as shown in Table II. The induction periods until the onset of primary nucleation, however, were different for the two specimens. PBSU generally appeared first in the lower experimental temperature range and vice versa in the higher range. Nonetheless, the two components show simultaneous spherulitic growth in any case under almost all the present experimental conditions. After the spherulites of one component emerge, the increase of the concentration of the other specimen in the residual melt suppresses the crystallization of it. Though this possibly explains the complex $T_{\mathrm{c}}$ dependence of $G$ of PVDCVC in Figure 2, the details are still under study.

Interpenetration is now discussed here. The microscopic results showed that the area of S2 became brighter from the lower left in Figure 3(a)-(c) and that the apparent birefringent pattern in $\mathrm{S} 2$ was almost the same with that of a PBSU spherulite in Figure 3(d). The
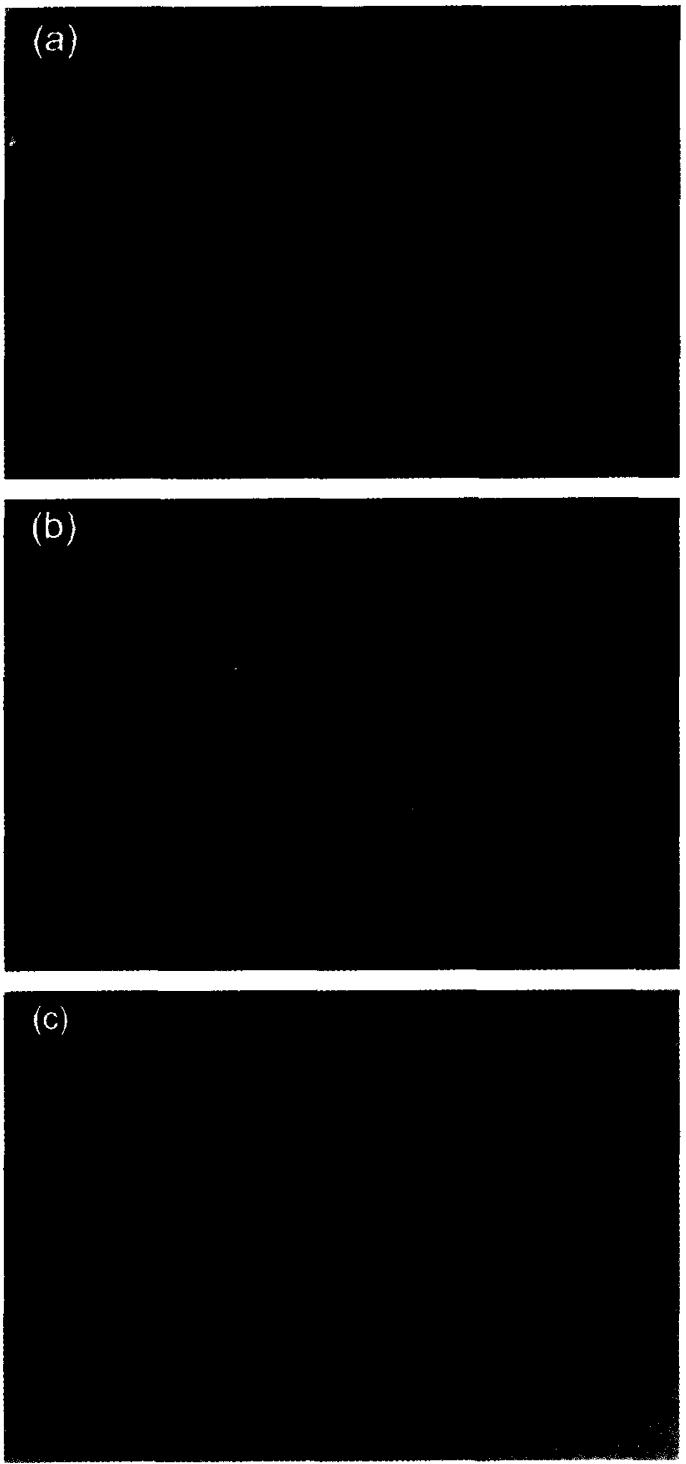

Figure 4. Birefringent patterns of PBSU spherulites. (a) Negative spherulites of neat PBSU crystallized at $368 \mathrm{~K}$ for $6 \mathrm{~min}$. (b) A $60 / 40$ blend crystallized at $358 \mathrm{~K}$ for $35 \mathrm{~min}$. (c) A $50 / 50$ blend crystallized at $363 \mathrm{~K}$ for $72 \mathrm{~min}$. Bar $100 \mu \mathrm{m}$.

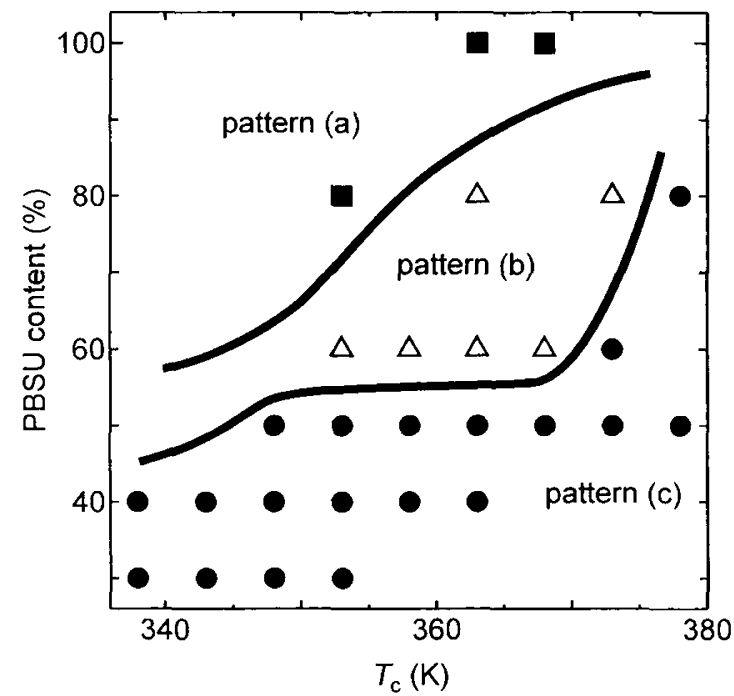

Figure 5. Dependence of the birefringent patterns of PBSU spherulites on $T_{\mathrm{c}}$ and the PBSU content. $\square$ : pattern (a), $\triangle$ : pattern (b), and : pattern (c) in Figure 4. 

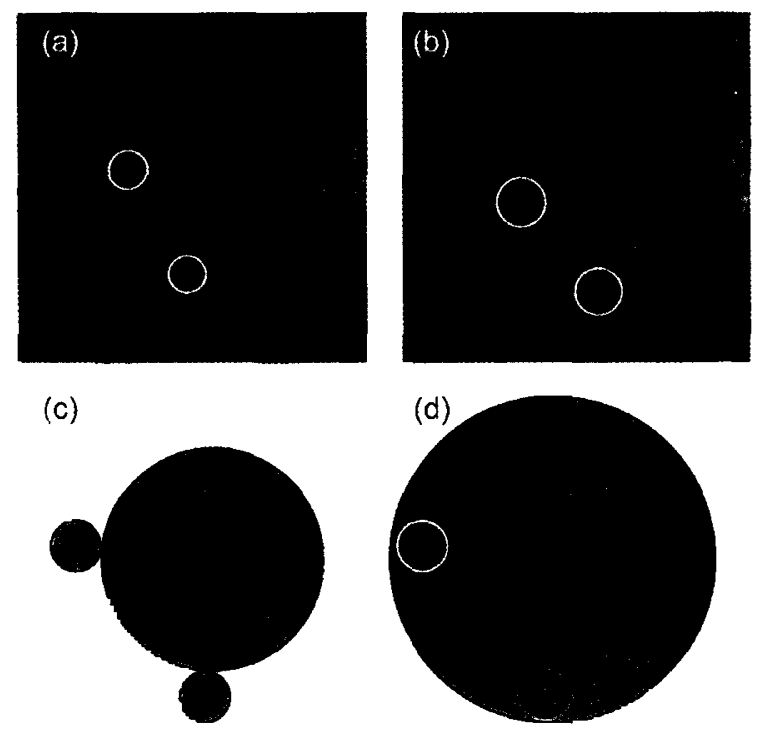

Figure 6. Penetration process of a PBSU spherulite of pattern (c) in Figure 4 for PBSU/PVDCVC $=\mathbf{5 0 / 5 0}$ crystallized at $363 \mathrm{~K}$ for (a) $45 \mathrm{~min}$ and (b) $65 \mathrm{~min}$. The white lines indicate the positions of PVDCVC spherulites. (c) and (d) are the schematic figures of (a) and (b), respectively. Bar $20 \mu \mathrm{m}$.

increase of brightness in S2 indicates that PBSU also crystallizes in this area when the difference in intrinsic birefringence between PBSU and PVDCVC is taken into account. Two possibilities are considered for these results. One is the penetration of S1 into S2 and the other is the superposition of the two spherulites in the film. Penetration of PBSU lamellae of S1 along PVDCVC lamellae in S2 describes the obtained results, especially the apparent birefringence of S2 in Figure 3(d). If S1 and S2 merely superposed on each other in a film, the observed image would be the superposition of the birefringent patterns of S1 and S2. However, it was never observed. The birefringent pattern in S2 in Figure 3 therefore proves the interpenetration in PBSU/PVDCVC blends. The termination of the growth of S2 after penetration, namely the difference in the size between $\mathrm{S} 2$ and S3, also denies the superposition of S1 and S2; S2 would probably continue to grow as S3 if it were superposed with $\mathrm{S} 1$. The change of apparent birefringence of PVDCVC in Figure 6 also indicates the penetration of the lamellae of PBSU along those of PVDCVC.

The results show that PBSU penetrates into PVDCVC and that PVDCVC does not penetrate into PBSU. This can be explained by assuming the low density of lamellae in PVDCVC spherulites. The low density causes the observed low birefringence of the PVDCVC spherulites. Since PVDCVC is a copolymer, its crystallinity is low as indicated by small DSC melting peaks. ${ }^{1}$ The situation of penetration is schematically illustrated in Figure 7. Since the spherulites fill the whole space in these blends, the residual amorphous substances of the constituents stay inside the spherulites, namely interlamellar and interfibrillar regions. The sparse spherulites of PVDCVC contain a sufficient amount of amorphous PBSU. This enables the PBSU spherulite to penetrate into PVDCVC spherulites. To confirm the difference in the lamellar density, we applied atomic force microscopy to the present system. It showed the direct evidences for

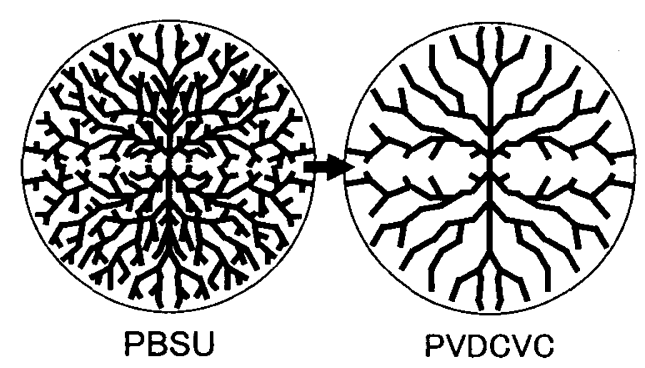

Figure 7. Schematic figure of the lamellar densities in PBSU and PVDCVC spherulites.

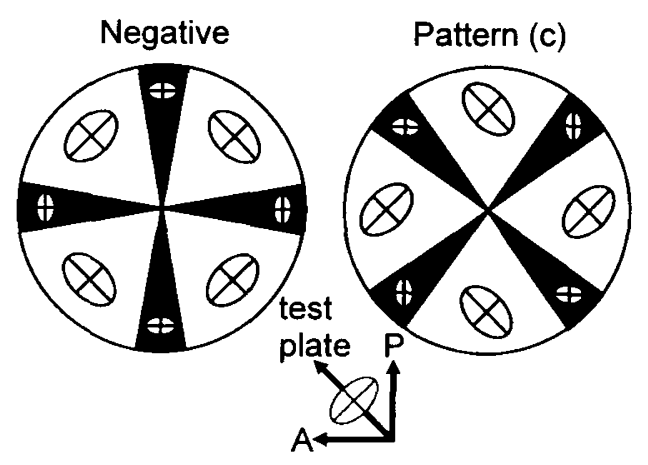

Figure 8. Schematic figure of the birefringent ellipsoids of PBSU in a negative spherulite and pattern (c) in Figure 4.

the difference in lamellar density of the two specimens. The detailed results will be published soon.$^{10}$

Figure 4(c) shows PBSU spherulites that is neither positive nor negative. This pattern indicates the birefringent ellipsoids uniformly incline as shown in Figure 8. The reason for this phenomenon is now under study.

Here, the present results are compared with the previous results ${ }^{1}$ where $M_{w}$ of PBSU was lower, 54000. In the previous study, interpenetrated spherulites were observed only under restricted conditions, PBSU/PVDCVC $\simeq 40 / 60$ and $T_{\mathrm{c}} \simeq 360 \mathrm{~K}$. They were observed, however, under almost all the experimental conditions in the present study as shown in Table II. This disagreement is ascribed to the difference in $G$ of PBSU. The value of $G$ in the present work was slower; for instance, it was about a tenth of what was reported in the previous study for PBSU/PVDCVC $\simeq 40 / 60$ and $T_{\mathrm{c}} \simeq 350 \mathrm{~K}$. The $M_{w}$ dependence of $G$ of PBSU is an interesting issue and requires more investigation.

Acknowledgment. This research was partly supported by Grant-in-Aid for Scientific Research of Ministry of Education, Science, Sports and Culture, Japan (No. 09450356). The authors would like to thank Showa Denko and Asahi Kasei for providing us of PBSU and PVDCVC, respectively. We are also grateful to Dr. J.-C. Lee, Mr. H. Tazawa, Ms. Y. Kosaka, and Mr. T. Tazawa for their help to the present work. 


\section{REFERENCES}

1. J.-C. Lee, H. Tazawa, T. Ikehara, and T. Nishi, Polym. J., 30, 780 (1998)

2. J.-C. Lee, H. Tazawa, T. Ikehara, and T. Nishi, J. Appl. Poly. Sci., B, Polym. Phys., 35, 2645 (1997).

3. J.-C. Lee, H. Tazawa, T. Ikehara, and T. Nishi, Polym. J., 30 327 (1998).

4. J. P. Penning and R. St. John Manley, Macromolecules, 29, 77
(1996).

5. J. P. Penning and R. St. John Manley, Macromolecules, 29, 84 (1996).

6. K. Fujita and T. Kyu, Macromolecules, 29, 91 (1996).

7. M. Aubin, Y. Bedard, M. F. Morrissette, and R. E. Prud' homme, J. Polym. Sci., B, Polym. Phys., 21, 233 (1983).

8. H. Zhang, and R. E. Prud'homme, J. Polym. Sci., B, Polym. Sci., 24, 723 (1987)

9. M. Avella and E. Martuscelli, Polymer, 29, 1731 (1988).

10. Y. Terada, T. Ikehara, and T. Nishi, in preparation. 